\title{
Die Prognosegüte von Wahlbörsen und Meinungsumfragen zur Bundestagswahl 2005
}

\author{
Lena-Maria Schaffer / Gerald Schneider
}

Die Gegenüberstellung von Wahlbörsenresultaten und Meinungsumfragen gehört zu den Ritualen, die in der Berichterstattung über Wahlen nach Bekanntgabe der Resultate einsetzen. Doch viele dieser Analysen beziehen sich nicht aufeigentliche Vorhersagen. In diesem Aufsatz stellen wir einen ähnlichen Vergleich an, beziehen uns aber aufPrognosen, die wir vor dem Wahlausgang für die Bundestagswahl 2005 errechneten. Die Analyse zeigt, dass die Wahlbörse Wahl\$treet auch 2005 besser abschnitt als die kommerziellen Institute. Angesichts der außergewöhnlich großen Prognosefehler besonders der Umfrageinstitute diskutieren wir überdies im Licht der Social Choice-Theorie, welche Auswirkungen fehlerhafte Umfragen auf Wablentscheidungen haben.

Vom Wahrsagen lässt sich's wohl leben in der Welt, aber nicht vom Wahrheit Sagen.

Georg Christoph Lichtenberg, Sudelbücher

\section{Einleitung}

Entgegen der Erwartung Lichtenbergs ist heute in vielen sozialwissenschaftlichen Disziplinen der Einsatz von Prognosen selbstverständlich. Auch die Politikwissenschaft setzt zunehmend analytische Techniken ein, um einzelne politische Ereignisse wie die $\mathrm{Zu}$ kunft Hongkongs (Bueno de Mesquita et al. 1985), Entscheidungsprozesse in der Europäischen Union (Bueno de Mesquita/Stokman 1994; Thomson et al. 2006), das zwischenstaatliche Konfliktrisiko (Beck et al. 2000) oder den Verlauf von bewaffneten Auseinandersetzungen (Schneider 2005) zu prognostizieren.

Im deutschsprachigen Raum verhalten sich Sozialwissenschaftler gegenüber Prognosen immer noch zögerlich. Dies gilt auch für die Wahlforschung. Während in den USA, Frankreich oder Großbritannien führende Zeitschriften Sonderausgaben zur Vorhersage einzelner Wahlausgänge publizieren, ist die Zahl systematischer Prognosen zum Ausgang von Bundestags- oder Landtagswahlen überaus spärlich. Eine Ausnahme sind die Veröffentlichungen zum Prognosemodell von T. Gschwend und H. Norpoth (z.B. Gschwend/Norpoth 2001; Norpoth/Gschwend 2005). Unsere Studie schließt sich diesen Bemühungen an und vergleicht, wie weit Meinungsumfragen und Wahlbörsen vom

1 Unser Dank gilt dem Team von ECCE TERRAM für die Bereitstellung der Wahl\$treet-Daten und Ingmar Nolte für seine Hilfe mit den ökonometrischen Schätzungen. 
tatsächlichen Wahlergebnis entfernt lagen. Dazu haben wir vor dem 18.9.2005 aufgrund der Börse „Wahl\$treet“ den möglichen Ausgang der Wahlen geschätzt. Diese out-of-Sample-Prognosen haben wir der Redaktion der PVS am 17.9.2005 zur Verfügung gestellt, sodass eine nachträgliche Anpassung unserer Vorhersagen an die Realität ausgeschlossen ist.

Unser Kurzartikel kann natürlich nicht das Manko an systematischen Wahlprognosen beheben. Aber er kann die Diskussion über kommerzielle Meinungsumfragen versachlichen helfen, die nach dem 18.9.2005 heftiger Kritik ausgesetzt waren. Zu unserem Erstaunen erfasste diese Debatte zunächst nicht den eigentlich fragwürdigen Aspekt von Prognosefehlern. Irrige Umfragen können das Wahlergebnis insofern verfälschen, als sie beispielsweise Unionsanhänger im Sinne des Split Ticket-Voting dazu verleiten, mit ihrer Zweitstimme den Freidemokraten zu helfen, da die Meinungsumfragen diese Partei eventuell entgegen der Realität als marginalisiert darstellen.

Bevor wir die normativen Implikationen falscher Prognosen diskutieren, wollen wir erstens klären, ob auch die deutsche Wahlbörse „Wahl\$treet“ im Jahr 2005 systematisch bessere Prognosen lieferte als die Meinungsumfragen. Natürlich ist eine solche einmalige Bestandsaufnahme nur begrenzt generalisierbar. Da die wissenschaftliche Auseinandersetzung mit dem Instrument der Wahlbörsen bis jetzt aber praktisch ausschließlich in ökonomischen Fachzeitschriften stattgefunden hat, erscheint uns eine Diskussion der Prognoseresultate gerechtfertigt. Zweitens erkunden wir, ob sich die Prognosegüte der Umfragen gegenüber der Wahlbörse im Zeitverlauf annähert, wie die theoretischen Forschung in diesem Bereich suggeriert (Kou/Sobel 2004). Drittens soll demonstriert werden, dass einfache Zeitreihenmodelle relativ präzise Prognosen erlauben. Wir präsentieren die Ergebnisse nach einer kursorischen Literaturübersicht.

\section{Vergleich von Prognosen: Theorie und Bewertungskriterien}

Nachdem es Ende der 1980er Jahre dem Iowa Presidential Stock Market, dem ersten vollelektronischen politischen Aktienmarkt, gelungen war, den Gewinner der US-Präsidentschaftswahlen 1988 erfolgreich vorherzusagen, wurden Wahlbörsen zu einem intensiv bearbeiteten Feld. Besonders beliebt ist es, die Prognosegüte dieses Instrumentes zu evaluieren und mit den Umfrageresultaten zu vergleichen. Unsere Analyse reiht sich in diese Forschungstradition ein. Vorausgeschickt sei allerdings, dass der in den meisten Fällen beobachtbare Erfolg der Wahlbörsen keineswegs überrascht. So ist aufgrund der Informationstheorie zu erwarten, dass Wahlbörsen in einem hohen Maße „informationseffizient" sind. Dies bedeutet, dass die Händler in ihren Entscheidungen alle relevanten Informationen berücksichtigen, da Fehlentscheidungen kostenträchtig sind (Kou/Sobel 2004). Zu diesen wesentlichen Informationen gehören neben den wichtigeren Wahlkampfereignissen natürlich auch Umfrageresultate, über die die Medien regelmäßig und oft in großer Aufmachung berichten. Entscheidend für den Vorhersageerfolg der Wahlbörsen ist ebenso, dass Meinungsforschungsinstitute lediglich ein Stimmungsbild der aktuellen Wahlabsicht in der Bevölkerung liefern. Nichts desto trotz stellt die Publizistik solche Stimmungsbilder regelmäßig als Prognosen über den Wahlausgang dar. Diese Vorhersagen sollten nach Kou und Sobel (2004) umso genauer sein, 
je näher der tatsächliche Wahltag liegt. Händler in einem politischen Aktienmarkt richten ihre Erwartungen jedoch auf den Wahltag aus. Deshalb ist zu erwarten, dass die Prognosen einer Wahlbörse eine geringere Varianz aufweisen als Umfragen, in denen aktuelle Ereignisse die Tagesstimmung beeinflussen, ohne jedoch notwendigerweise eine längerfristige Wirkung zu entfalten.

Politische Aktienmärkte hat die experimentelle Ökonomie entwickelt, um die Effizienz von Märkten zu analysieren (Forsythe et al. 1992; Beckmann/Werding 1996). Eine solche Analyse hat Brüggelambert (2004) jüngst für vier deutsche Wahlbörsen vorgelegt. Er zeigt, dass in den 1990er Jahren die gut informierten Marktteilnehmer höhere Gewinne machen konnten als die weniger gut informierten Teilnehmer, wobei sich die Sympathie zugunsten einer Partei nicht negativ äußerte. Berlemann und Schmidt (2001) stellen in einer Meta-Evaluation ebenso fest, dass die Prognosefehler der Wahlbörsen auf ein Überbewerten der kleinen Parteien zurückzuführen ist. Hansen, Schmidt und Strobel (2004) konstatieren überdies für zwei Berliner Wahlbörsen, dass sich dieses Instrument politisch manipulieren lasse.

Brüggelambert (1999) sowie Berlemann und Schmidt (2001) haben systematisch Wahlbörsen und Umfragen miteinander verglichen. Das Problem solcher Vergleiche ist, dass sie nur beschränkt die Prognosemöglichkeiten nutzen, die Wahlbörsen eigen sind. So bezieht sich die Evaluation der Prognosegüte zumeist auf einen Vergleich der tatsächlichen Resultate mit dem letzten Umfragewert bzw. den vor Marktschluss realisierten Kursen an der Wahlbörse. Obwohl dieses Vorgehen wertvolle Informationen liefert, reicht es nicht aus. Wie Lewis-Beck (2005) zeigt, gehört zu einer wissenschaftlichen Vorhersage auch, dass sie tatsächlich in die Zukunft reicht und nicht einfach gerade erhältliche Gegenwartswerte als Prognosen verkauft. Deshalb ist aufgrund der vorhandenen Informationen zusätzlich zu berechnen, wie die Zustimmung zu einzelnen Parteien oder möglichen Koalitionen am tatsächlichen Wahltag ausfallen könnte.

Diese Studie berechnet deshalb aufgrund der realisierten Kurse an der Wahlbörse und zweier Wahlumfragen sog. „out-of-Sample“-Prognosen, d.h. Vorhersagen, die auf einen nicht erfassten Zeitpunkt verweisen. Um diesem Ideal nachzuleben, haben wir am Vortag der Wahl deshalb der PVS-Redaktion unsere Berechnungen zukommen lassen; die Daten, die in die Analyse eingingen, sind auf der Replikations-Homepage des zweiten Verfassers einsehbar. ${ }^{2}$ Anzumerken ist dabei, dass unser Prognosenvergleich einzig der Vorhersage und nicht der Erklärung dient. Mit anderen Worten: Unser Vorgehen ist atheoretisch und berücksichtigt nicht wie etwa die Studien von Gschwend und Norpoth substanzielle Variablen als Hilfsmittel, um die Prognose zu verbessern.

\section{Vorgehensweise und empirische Resultate}

Die ersten Wahlbörsen kamen in Deutschland schon in den frühen 1990er Jahren zum Einsatz. Einem breiteren Publikum ist besonders die Wahlbörse „Wahl\$treet" bekannt, die bei der Wahl der ersten Schröder-Regierung recht akkurate Kurswerte generierte. Nicht zuletzt aus diesem Grund wurde Wahl\$treet auch für den Wahlkampf 2005 von

2 http://www.uni-konstanz.de/FuF/Verwiss/GSchneider/downloads/daten.htm 
drei Zeitungen mit Unterstützung von ECCE TERRAM betrieben. Vom 25. Juli bis zum Wahltag konnten interessierte Händler für ihre eingebrachte Investition von 5 bis 50 Euro Aktienportfolios kaufen. Insgesamt tätigten ca. 2500 aktive Händler 184.433 Transaktionen über die Homepage (www.wahlstreet.de). Die „Wahl\$treet“-Zeitreihen beruhen auf drei täglichen Messzeitpunkten: 8:00, 16:00 und 24:00; für die Prognosemodelle beziehen wir uns auf die um 16:00 Uhr gültigen Kurse.

Abbildung 1: Abweichung der Meinungsforschungsinstitute von der Wahlbörse für Unterstützungswerte der SPD

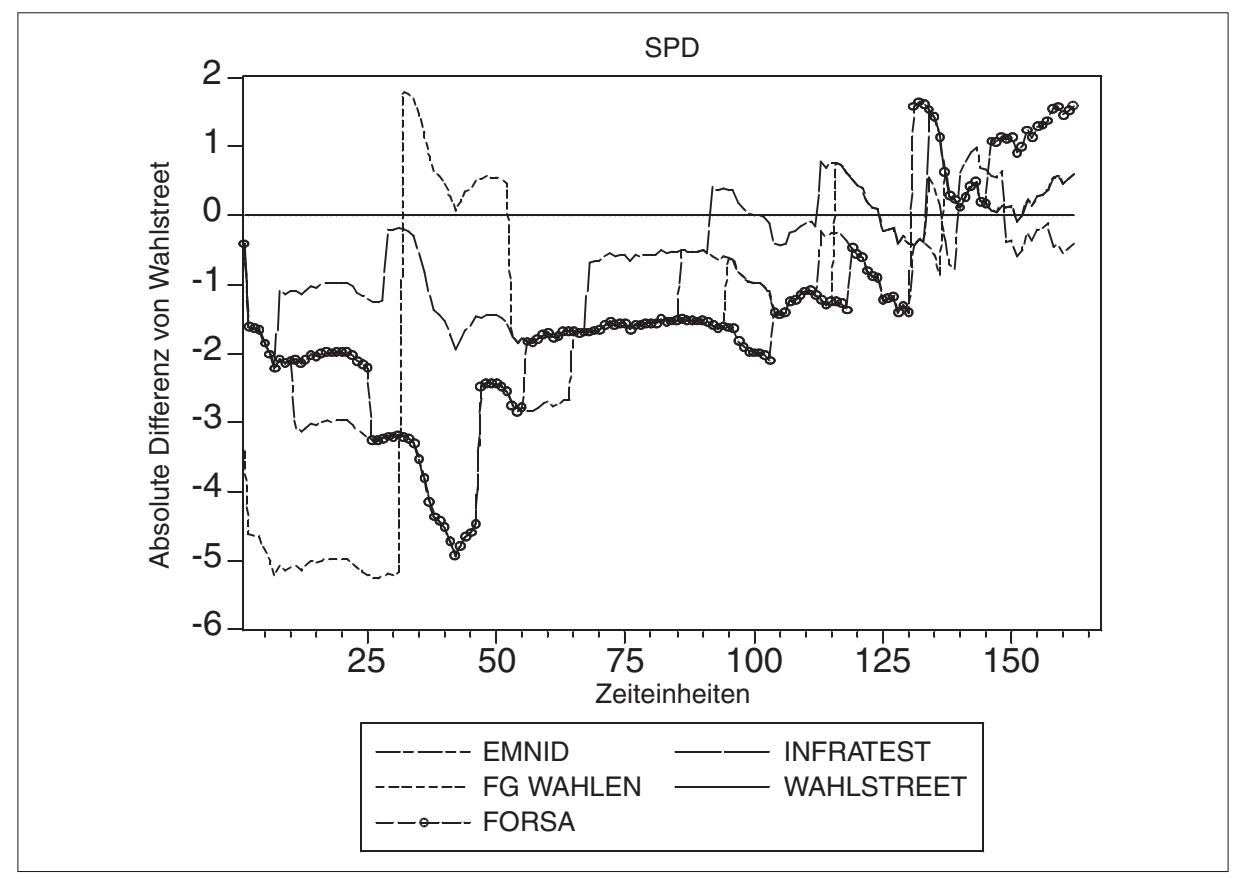

Die Abbildungen 1 und 2 zeigen, wie sich die Unterstützung bei dieser Wahlbörse, die jeweils auf 0 festgeschrieben ist, im Vergleich zu den Meinungsumfragen entwickelt hat. Da wir nicht nach einer umfassenden Evaluation sämtlicher Umfrageunternehmen streben, haben wir jene beiden Umfragen gewählt, die sich am 10.9. am stärksten von den Wahlbörsekursen unterschieden bzw. diesen am nächsten lagen. Dabei handelt es sich um die Prognosen der Umfrageinstitute Forsa und Emnid.

Besonders deutlich ist in Abbildung 1 die abnehmende Varianz für die SPD zu erkennen. Über den Zeitraum von 56 Tagen mit drei täglichen Messpunkten lässt sich eine Konvergenz von Wahlbörse und kommerziellen Umfragen erkennen. Auch in Abbildung 2 ist eindeutig ein Trend hin zu den an der "Wahl\$treet“ stets tiefer liegenden Unterstützungswerten für die CDU zu sehen.

Um die Prognosegüte der einzelnen Reihen bewerten zu können, vergleichen wir die Wahlergebnisse mit prognostizierten Werten. Sämtliche Prognosen beruhen auf demselben ARMA $(1,1)$-Grundmodell und damit einem Zeitreihenverfahren, das aus 
Abbildung 2: Abweichung der Meinungsforschungsinstitute von der Wahlbörse für Unterstützungswerte der CDU

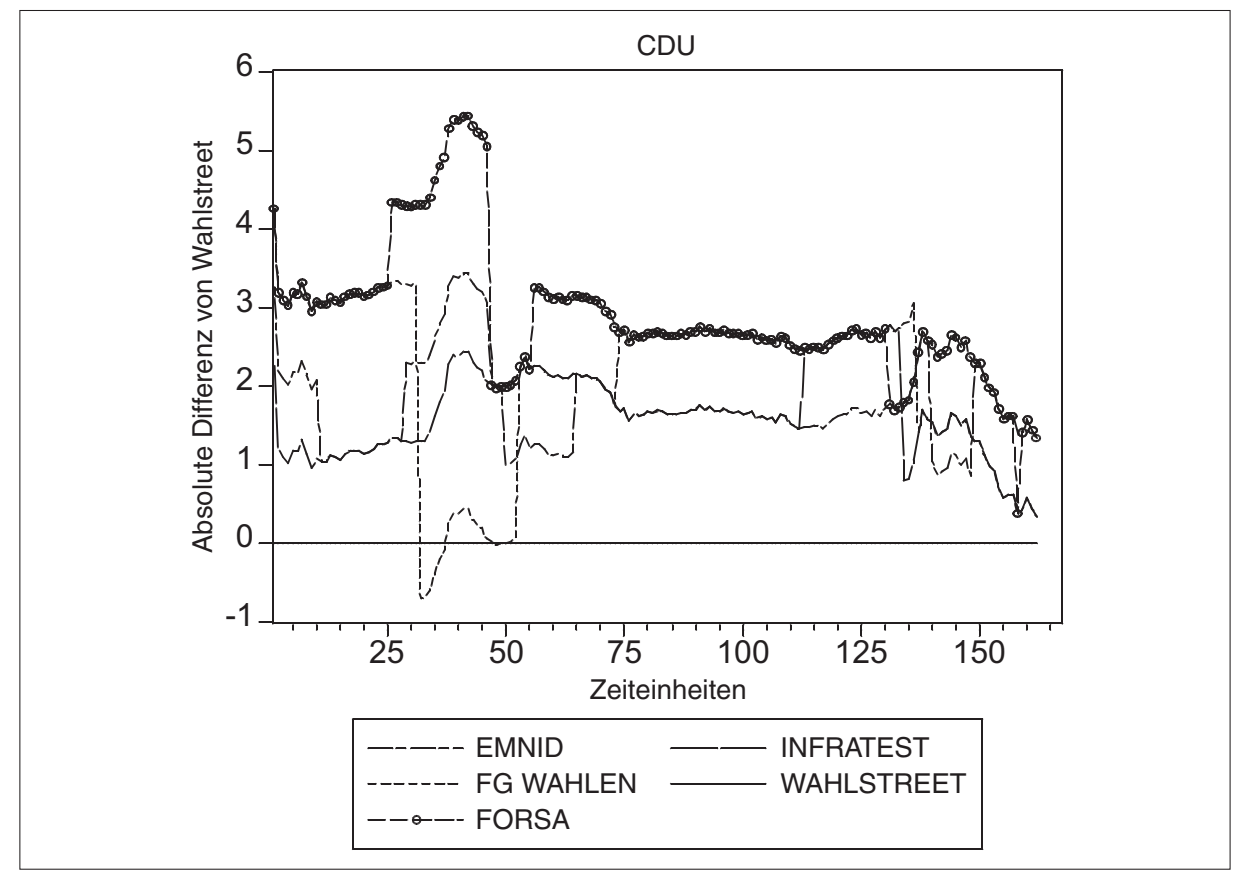

einem autoregressiven und einem Moving Average-Parameter im Fehlerterm mit einer Zeitverzögerung von jeweils einer Zeiteinheit besteht (vgl. Enders 2004). Bei allen Zeitreihen mit Ausnahme der Berechnungen für die Wahlbörsen-Werte der Unterstützung für die SPD ist kein klarer Trend erkennbar. Um die Berechnungen vergleichbar zu halten, haben wir aber auf eine Differenzierung der SPD-Wahlbörsenwerte verzichtet, über die sich diese Reihe stationär machen ließe. Die Varianz, die mit diesen einfachen Modellen erklärt werden kann, schwankt zwischen 96 und 64 Prozent. ${ }^{3}$

Tabelle 1 zeigt den Prozentanteil der Stimmen, welche die Parteien tatsächlich erhielten und welche die Umfragen und die Wahlbörse zu verschiedenen Zeitpunkten nahe legten. Wir präsentieren Umfrageresultate und Wahlbörsenkurse vom 10. und vom 17. September 2005. Für die Wahlbörse haben wir für beide Termine out-ofSample-Prognosen errechnet. Der Prognosehorizont unterscheidet sich, je nachdem, welches Stichdatum wir wählten: So beträgt dieser sog. „Lead“ bei den Daten für den 10.9.2005 acht Tage; beim 17.9.2005 reicht die Prognose eine Einheit über die Zahl der Kurswerte hinaus.

Tabelle 1 bestätigt, dass sich in einem zunehmend fragmentierten Mehrparteiensystem wie in Deutschland mit Wahlbörsen auch 2005 präzisere Resultate errechnen ließen als mit Hilfe von kommerziellen Umfragen. So liegt der Prognosefehler für die ARMA-Modelle im Schnitt unter den Prognosefehlern für die zwei Umfrageinstitute.

3 Kompliziertere Modelle, die bis zu sieben autoregressive Lags berücksichtigten, verbesserten die Prognosekraft nicht merklich. 
Tabelle 1: Wahlergebnis, realisierte Kurse und Umfragewerte sowie Wahlprognosen (Parteien)

\begin{tabular}{|c|c|c|c|c|c|c|c|c|}
\hline & $\begin{array}{l}\text { Ergebnis } \\
\text { BTW } \\
18.09 .05\end{array}$ & $\begin{array}{l}\text { Wahlbörse } \\
10.09 .05\end{array}$ & $\begin{array}{l}\text { Wahlbörse } \\
10.09 .05 \\
\text { (Pro- } \\
\text { gnose) }\end{array}$ & $\begin{array}{c}\text { Wahlbörse } \\
17.09 .05\end{array}$ & $\begin{array}{l}\text { Wahlbörse } \\
17.09 .05 \\
\text { (Pro- } \\
\text { gnose) }\end{array}$ & $\begin{array}{l}\text { Wahlbörse } \\
18.09 .05 \\
\text { (Schluss- } \\
\text { kurs) }\end{array}$ & $\begin{array}{c}\text { Emnid } \\
13.09 .05 \\
\text { (letzte } \\
\text { Umfrage) }\end{array}$ & $\begin{array}{c}\text { Forsa } \\
16.09 .05 \\
\text { (letzte } \\
\text { Umfrage) }\end{array}$ \\
\hline SPD & 34,3 & 33,58 & $35,32^{1}$ & 33,41 & $33,51^{6}$ & 33,11 & 33,5 & 33,0 \\
\hline CDU & 35,2 & 39,59 & $40,22^{2}$ & 40,66 & $40,39^{7}$ & 39,99 & 42,0 & 42,0 \\
\hline Grüne & 8,1 & 8,14 & $8,11^{3}$ & 8,01 & $8,06^{8}$ & 7,84 & 7,0 & 6,5 \\
\hline FDP & 9,8 & 7,40 & $7,48^{4}$ & 7,30 & $7,34^{9}$ & 9,70 & 6,5 & 7,5 \\
\hline Linkspartei & 8,7 & 8,43 & $9,57^{5}$ & 7,75 & $8,53^{10}$ & 7,68 & 8,0 & 7,5 \\
\hline $\begin{array}{l}\varnothing \text { quadrier- } \\
\text { ter Fehler }\end{array}$ & & 5,13 & 6,48 & 7,55 & 6,72 & 5,10 & 11,89 & 11,44 \\
\hline
\end{tabular}

Angaben: Die Ergebnisse wurden mit Hilfe des Computerprogramms EViews 5.0 errechnet. Die Wahlergebnisse schließen den Wahlkreis 160 (Dresden I) nicht ein.

${ }^{1}$ adjusted $\mathrm{R}^{2}=0.95$, Durbin-Watson $=1.93$, Schwarz criterion $=0.35 ;^{2}$ adjusted $\mathrm{R}^{2}=0.96$, Durbin-Watson $=1.92$, Schwarz criterion $=0.33 ;{ }^{3}$ adjusted $\mathrm{R}^{2}=0.80$, Durbin-Watson $=2.06$, Schwarz criterion $=-0.53 ;{ }^{4}$ adjusted $\mathrm{R}^{2}=0.74$, Durbin-Watson $=2.36$, Schwarz criterion $=$ $-0.19 ;{ }^{5}$ adjusted $\mathrm{R}^{2}=0.64$, Durbin-Watson $=1.88$, Schwarz criterion $=-3.77 ;{ }^{6}$ adjusted $\mathrm{R}^{2}=$ 0.84 , Durbin-Watson $=1.91$, Schwarz criterion $=-3.75 ;{ }^{7}$ adjusted $\mathrm{R}^{2}=0.77$, Durbin-Watson $=$ 1.89 , Schwarz criterion $=-2.65 ;{ }^{8}$ adjusted $\mathrm{R}^{2}=0.67$, Durbin-Watson $=2.11$, Schwarz criterion $=$ $-2.20 ;{ }^{9}$ adjusted $\mathrm{R}^{2}=0.89$, Durbin-Watson $=2.72$, Schwarz criterion $=0.58 ;{ }^{10}$ adjusted $\mathrm{R}^{2}=$ 0.90 , Durbin-Watson $=2.58$, Schwarz criterion $=0.76$.

Da sämtliche Institute der CDU zuletzt eine Stärke von 42 und mehr Prozent prognostizierten, liegt der Fehler der Meinungsforscher weit über den aus der Wahlbörse abgeleiteten Werten. Für den gesamten Zeitraum hatte die „Wahl\$treet“ für die Union zwischen 39,31 (Tiefststand am 9. September) und 40,98 Prozent (Höchststand am 26. Juli) prognostiziert. Eine mögliche Erklärung, warum auch „Wahl\$treet“ für 2005 trotz vergleichsweise besserer Prognose relativ weit vom Ergebnis entfernt lag, könnte mit der Beeinflussung der Händler durch Umfrageergebnisse zusammenhängen. Auch wenn einige Forschungsartikel hier keinen Zusammenhang finden (z.B. Forsythe 1992), so scheint es doch plausibel, dass Händler durch die Persistenz der Umfragewerte auf über 40 Prozent für die CDU ihre Erwartungen dementsprechend anglichen. Eine weitergehende Untersuchung des Händlerverhaltens auf Individualebene könnte hier wertvolle Erkenntnis bringen.

Weiter lässt sich in Tabelle 1 erkennen, dass die Abschlusskurse von „Wahl\$treet“ am Wahltag die geringsten Abweichungen vom Ergebnis aufweisen. Da die Händler in den letzten zwei Tagen keine Informationen aus den Umfragen mehr beziehen konnten, ist der Gewinn an Prognosegüte deshalb auf die Verarbeitung anderer Informationen zurückzuführen. 


\section{Schluss}

Die Bundestagswahl 2005 wird wohl in die Geschichte der kommerziellen Meinungsforschung als Schlüsselereignis eingehen: Während sich bei anderen Wahlkämpfen aus den Umfragen relativ akkurat die Gewinner und Verlierer vorhersagen ließen, erlitt die Umfrageforschung ein Prognosedesaster. Sicherlich ist die Güte der Vorhersagen, die wir aus der Börse "Wahl\$treet" mit Hilfe von Zeitreihenmodellen atheoretisch ableiteten, ebenfalls nicht berauschend. Aber dennoch sollte es der kommerziellen Umfrageforschung zu denken geben, dass ihr prognostizierter Wahlausgang noch weiter weg von der Realität lag.

Die fehlende Genauigkeit hat Auswirkungen für die akademische Forschung, die beispielsweise Umfrage-Zeitreihen als Input für die Berechnung von Popularitätsfunktionen verwendet. Während die Entwicklung der Regierungsunterstützung als Funktion von substanziellen ökonomischen und politischen Variablen gewertet wird, deutet unsere Analyse darauf hin, dass die Messfehler größer sind, als die empirische Forschung bislang annahm. Wie systematisch die Abweichungen sind, wäre eine Untersuchung wert. Die Umfragen lassen sich wohl nur verbessern, wenn die theoretischen Grundlagen, auf denen die Adjustierungen der Umfrageergebnisse beruhen, zunächst einmal einer kritischen Überprüfung unterzogen werden.

Am wichtigsten sind aber die staatspolitischen Implikationen, die gravierende Umfragefehler haben. So können sich Wähler auf der Grundlage von irreführenden Umfrageergebnissen zur taktischen Stimmabgabe verleitet sehen, um eine Partei zu stützen oder zu bestrafen, obwohl dies gar nicht der wahren Präferenz entspricht. Damit vergrößert sich die Gefahr, dass die kollektiven Wahlentscheidungen die wahren Intentionen der Wähler nicht ausreichend abbilden. Ähnliche Verzerrungen zwischen manifester und tatsächlicher Präferenz sind ein Standardthema der Social Choice-Forschung seit Arrow (1963). Bis jetzt fehlt es an Untersuchungen, inwiefern fehlerhafte Informationen die Anomalien verstärken, die demokratische Wahlen charakterisieren. Da Wahlbörsen geringere Fehler aufweisen, scheint es sich aufzudrängen, dieses Instrument in der Berichterstattung über einen Wahlkampf vermehrt zu beachten. Dies gilt auch dann, wenn durch die stärkere mediale Aufmerksamkeit die Wahrscheinlichkeit der gezielten Manipulation von Wahlbörsen durch Parteigänger steigt (Hansen/Schmidt/Strobel 2004). Da über die zunehmende Resonanz auch die Zahl der Markteilnehmer wachsen wird, lässt sich diese Gefahr jedoch relativieren.

\section{Literatur}

Arrow, Kenneth J., 1963: Social Choice and Individual Values. New York.

Beck, Nathaniel/King, Gary/Zeng, Langche, 2000: Improving Quantitative Studies of International Conflict, in: American Political Science Review 94, 21-36.

Beckmann, Klaus/Werding, Martin, 1996: „Passauer Wahlbörse“: Information Processing in a Political Market Experiment, in: Kyklos 49, 171-204.

Berlemann, Michael/Schmidt, Caarsten, 2001: Predictive Accuracy of Political Stock Markets: Empirical Evidence from an European Perspective. Dresdner Beiträge zur Volkswirtschaftslehre Nr. $5 / 01$. 
Brüggelambert, Gregor, 1999: Institutionen als Informationsträger. Erfahrungen mit Wahlbörsen. Marburg.

Brüggelambert, Gregor, 2004: Information and Efficiency in Political Stock Markets. Using Computerized Markets to Predict Election Results, in: Applied Economics 36, 753-768.

Bueno de Mesquita, Bruce/Newman, David/Rabushka, Alvin, 1985: Forecasting Political Events. The Future of Hong Kong. New Haven.

Bueno de Mesquita, Bruce/Stokman, Frans (Hrsg.), 1994: European Community Decision Making. Models, Applications, and Comparisions. New Haven.

Enders, Walter, 2004: Applied Econometric Time Series. Hoboken.

Forsythe, Robert/Nelson, Forrest/Neumann, George R./Wright, Jack, 1992: Anatomy of an Experimental Political Stock Market, in: American Economic Review 82, 1142-1161.

Gschwend, Thomas/Norpoth, Helmuth, 2001: Wenn am nächsten Sonntag...: Ein Prognosemodell für Bundestagswahlen, in: Klingemann, Hans-Dieter/Kaase, Max (Hrsg.), Wahlen und Wähler. Analysen aus Anlass der Bundestagswahl 1998. Wiesbaden, 473-499.

Hansen, Jan/Schmidt, Carsten/Strobel, Martin, 2004: Manipulation in Political Stock Markets - Preconditions and Evidence, in: Applied Economic Letters 11, 459-463.

Kou, Steven G./Sobel, Michael E., 2004: Forecasting the Vote: A Theoretical Comparison of Election Markets, in: Political Analysis 12, 277-295.

Lewis-Beck, Michael S., 2005: Election Forecasting: Principles and Practice, in: British Journal of Politics and International Relations 7, 145-164.

Norpoth, Helmut/Gschwend, Thomas, 2005: Mit Rot-Grün ins Schwarze getroffen: Prognosemodell besteht Feuertaufe, in: Falter, Jürgen W./Gabriel, Oscar W./Weßels, Bernhard (Hrsg.), Wahlen und Wähler. Analysen aus Anlass der Bundestagswahl 2002. Wiesbaden.

Schneider, Gerald, 2005: Banking on Broking: Forecasting Conflict in the Levant With Financial Data. Paper für die Konferenz Illuminating the Shadow of the Future, Ann Arbor, MI, 23.-25. September 2005.

Thomson, Robert/Stokman, Frans/Achen, Christopher/König, Thomas (Hrsg.), 2006 (i.E.): The European Union Decides. Cambridge: Cambridge University Press. 